\title{
Correction to: Copyright Volume 31, Issues 1-4, Acta Mechanica Solida Sinica
}

\author{
Li Shen ${ }^{1 \star}$ \\ $\left({ }^{1}\right.$ Rm.408-412, Beijing Ideal Plaza, No. 58 Northwest, 4th Ring Road, Haidian District, Beijing 100080, \\ China)
}

published online 22 November 2018

(C) The Chinese Society of Theoretical and Applied Mechanics 2018

Correction to: https://doi.org/10.1007/s10338-018-0001-2
https://doi.org/10.1007/s10338-018-0012-z
https://doi.org/10.1007/s10338-018-0011-0
https://doi.org/10.1007/s10338-018-0004-z
https://doi.org/10.1007/s10338-018-0005-y
https://doi.org/10.1007/s10338-018-0002-1
https://doi.org/10.1007/s10338-018-0003-0
https://doi.org/10.1007/s10338-018-0008-8
https://doi.org/10.1007/s10338-018-0010-1
https://doi.org/10.1007/s10338-018-0022-x
https://doi.org/10.1007/s10338-018-0019-5
https://doi.org/10.1007/s10338-018-0014-x
https://doi.org/10.1007/s10338-018-0006-x
https://doi.org/10.1007/s10338-018-0020-z
https://doi.org/10.1007/s10338-018-0009-7
https://doi.org/10.1007/s10338-018-0007-9
https://doi.org/10.1007/s10338-018-0026-6
https://doi.org/10.1007/s10338-018-0018-6
https://doi.org/10.1007/s10338-018-0021-y
https://doi.org/10.1007/s10338-018-0025-7
https://doi.org/10.1007/s10338-018-0015-9
https://doi.org/10.1007/s10338-018-0016-8
https://doi.org/10.1007/s10338-018-0017-7
https://doi.org/10.1007/s10338-018-0027-5
https://doi.org/10.1007/s10338-018-0013-y
https://doi.org/10.1007/s10338-018-0038-2
https://doi.org/10.1007/s10338-018-0031-9
https://doi.org/10.1007/s10338-018-0029-3
https://doi.org/10.1007/s10338-018-0040-8
https://doi.org/10.1007/s10338-018-0023-9
https://doi.org/10.1007/s10338-018-0024-8
https://doi.org/10.1007/s10338-018-0032-8
https://doi.org/10.1007/s10338-018-0035-5
https://doi.org/10.1007/s10338-018-0028-4
https://doi.org/10.1007/s10338-018-0037-3

In all the articles in Acta Mechanica Solida Sinica, Volume 31, Issues 1-4 the copyright is incorrectly displayed as "The Chinese Society of Theoretical and Applied Mechanics and Technology" where it should be "The Chinese Society of Theoretical and Applied Mechanics".

\footnotetext{
* Corresponding author. E-mail: li.shen@springer.com
} 